\title{
Minimalinvasive versus offene Chirurgie bei Malignomen des Gastrointestinaltrakts
}

\author{
Gesprächsleiter: Hubertus Feußner (München) Jürgen Weitz (Dresden) \\ Teilnehmer: \\ Claus-Dieter Heidecke (Greifswald) Tobias Keck (Lübeck) \\ Wolfram Lamadé (Überlingen) Norbert Senninger (Münster)
}

\author{
Frage 1: In der Anfangszeit der laparoskopisch- \\ onkologischen Chirurgie waren die Port-Site- \\ Metastasierung und die allgemeine Tumorzell- \\ verschleppung ein Grund, vor der minimalinvasiven \\ Durchführung von Krebsoperationen zu warten. \\ Wie relevant ist das heute noch?
}

Heidecke: Port-Site-Metastasen sind selten; die Angst vor Port-Site-Metastasen ist weitgehend unbegründet. Nach meinem Literaturkenntnisstand treten Port-Site-Metastasen weiterhin deutlich erhöht bei Gallenwegskarzinomen auf. Daher operieren wir Gallenblasenkarzinome grundsätzlich noch offen.

Keck: Zur Frage von Trokarkanalmetastasen existieren vornehmlich experimentelle Studien. Durch die Anlage eines Pneumoperitoneums mit erhöhtem Druck und der Anwendung von $\mathrm{CO}_{2}$ sowie durch die laparoskopische Gewebemanipulation war suggeriert worden, dass die Inzidenz von Trokarmetastasen erhöht ist im Vergleich zu Implantationsmetastasen im Bereich offener Zugangswege. In diesen Studien, unter anderem von Lee et al. [1], zeigte sich im Tierexperiment, dass insbesondere die traumatische Manipulation eines Tumors zu mehr Port-Site-Metastasen führte als die Gruppe der atraumatischen Manipulation des Tumors. Wittich et al. [2] konnten in einer experimentellen Studie mit $\mathrm{CO}_{2}$-Laparoskopie zeigen, dass zur Aerosolisation von Tumorzellen eine immense Zahl intraabdomineller und intraperitonealer Tumorzellen notwendig ist. In dieser Risikokonstellation bei ausgedehnter Peritonealkarzinose ist auch die Metastasierung im Bereich konventioneller Zugangswege ein bekanntes Problem. In einer kumulativen Betrachtung von klinischen Daten, die zwischen 1996 und 2002 publiziert worden sind, zeigt sich, dass bei über 4000 Patienten die Inzidenz von Trokarmetastasen in der kolorektalen onkologischen Chirurgie nur $0,75 \%$ beträgt und daher heute keine relevante Rolle spielt.

Wichtige Voraussetzungen sind und bleiben die möglichst geringe direkte Traumatisierung des Tumors sowie der Einsatz von Bergefolien und Bergebeuteln in der onkologischen laparoskopischen Chirurgie.

Lamadé: Port-Site-Metastasen sind seltene Komplikationen der laparoskopischen Chirurgie und treten mit unter $0,1 \%$ nicht häufiger als bei offenen Operationen auf. Die Besorgnis stammt aus älteren Quellen, die in großen Serien nicht mehr reproduzierbar waren. Die Qualität und Gewebeschonung laparoskopischer Operationen sollte eher niedrigere Raten an Tumorzellverschleppung in die abdominellen Inzisionen erwarten lassen.

Senninger: Das war wahrscheinlich von Anfang an ein falscher Eindruck. Nachfolgende retrospektive Untersuchungen haben ja im Prinzip bei Trokarzugängen im Vergleich zur offenen Laparotomie kein erhöhtes Risiko dargestellt. Es ist anzunehmen, dass sowohl die Port-Site-Metastasen als auch die Metastasen in einer Laparotomiewunde nach offener Operation Ausdruck einer bereits bestehenden okkulten peritonealen Metastasierung sind und nichts mit der laparoskopischen Methode an sich zu tun haben.

\footnotetext{
Frage 2: Gibt es auch künftig «systemimmanente» Grenzen der laparoskopischen Technik, die nicht überwunden werden können, sodass bestimmte Indikationen immer der offen chirurgischen Vorgehensweise vorbehalten bleiben werden?
}

Heidecke: Systemimmanente Grenzen kaum, jedoch erscheinen kleine Tumoren, die nicht laparoskopisch gesehen

\begin{tabular}{ll}
\hline KARGER & @ 2013 S. Karger GmbH, Freiburg \\
Fax +497614520714 & Accessible online at: \\
Information@Karger.com & www.karger.com/vim \\
www.karger.com &
\end{tabular}


und/oder getastet werden und somit nicht sicher lokalisiert werden können, nicht sinnvoll laparoskopisch operabel. Ähnlich verhält es sich bei großen, wandüberschreitenden Tumoren, wo die Gefahr einer Tumoreröffnung und Tumorzellverschleppung besteht.

Keck: Systemimmanente Grenzen der laparoskopischen Technik beziehen sich zum Ersten vornehmlich auf Pathologien, die große Operationsfelder betreffen, und zum Zweiten auf Pathologien, die aufgrund der Größe des Tumors Bergeschnitte erfordern, die letztlich den Zugangswegen eines offenen Operationsverfahrens gleichkommen. Exemplarisch seien hierzu in der onkologischen Chirurgie die systematische parietale und viszerale Peritonektomie im Rahmen von HIPEC(hypertherme intraperitoneale Chemotherapie)-Verfahren erwähnt sowie die Resektionen im Sinne onkologischer Multiviszeralresektionen, wo die Vorzüge der Laparoskopie im onkologischen Setting schwer zu demonstrieren sein werden. Die bisher in manchen Bereichen von der hohen Expertise des Laparoskopikers abhängigen komplexen Rekonstruktionsverfahren, wie beispielsweise die laparoskopische Rekonstruktion nach total laparoskopischer Pankreatoduodenektomie, sind nach meiner subjektiven Ansicht keine systemimmanenten Grenzen in der laparoskopischen Technik, sondern werden gerade im Bereich komplexer rekonstruktiver Techniken durch Weiterentwicklung von Instrumentarien, bessere dreidimensionale Visualisierung und besseres laparoskopisches Nahtmaterial in der Zukunft keine relevanten Limitierungen der laparoskopischen Technik darstellen.

Lamadé: Grenzen sind zunächst nicht prinzipiell zu erkennen. Galt noch bis vor Kurzem eine perforierte Appendizitis als Kontraindikation für eine laparoskopische Operation, so ist sie heute Standard und in erfahrenen Händen sogar der offenen Operation überlegen, was Morbidität und Krankenhausaufenthalt betrifft. Tumoroperationen galten lange als nicht praktikabel. Mittlerweile sind T3-Tumoren des Kolons und Rektums in spezialisierten Kliniken als laparoskopische Operationen Routine. Die Studienlage bescheinigt mindestens eine gleiche onkologische Qualität.

Wahrscheinlich ist sie sogar der offenen Operation in vielerlei Hinsicht überlegen, da die Operation wegen der exzellenten Ausleuchtung und Vergrößerung auf kleinerem Raum wie im kleinen Becken weniger traumatisch und exakter ist. Die Anastomoseninsuffizienzrate ist in vielen Zentren auch bei der tiefen Rektumresektion so niedrig, dass die Vorschaltung eines Ileostomas nicht mehr grundsätzlicher Standard ist.

Die Adipositas galt bisher noch als Kontraindikation für laparoskopische Operationen, obwohl diese Patientengruppe als Hauptprofiteur minimalinvasiver Eingriffe angesehen wurde, da bei ihnen das Wundinfektionsrisiko dramatisch gesenkt werden würde. So konnten wir kürzlich zeigen, dass durch die geschickte Kombination verschiedener Techniken bei morbid adipösen Patienten $\left(\mathrm{BMI} \geq 50 \mathrm{~kg} / \mathrm{m}^{2}\right)$ Kolonresek- tionen in Hybrid-NOTES-Technik, d.h. ohne sichtbare Narben, möglich sind. Dies gelang selbst bei Operationen mit onkologischem Anspruch. Die Whipple'sche Operation als die Königsdisziplin der Viszeralchirurgie ist laparoskopisch in geübten Händen in Serie möglich.

Was der konventionellen Chirurgie bleibt, sind vorerst große Debulking-Operationen bei organüberschreitenden Tumoren oder massive Verwachsungsbäuche, wo die noch immer unübertroffenen taktilen Qualitäten der Finger einen enormen Zeitvorteil gegenüber der rein optisch navigierten laparoskopischen oder robotischen Operation verschaffen. Auch hier wird jedoch in Zukunft die Sensorik sicher weitere Innovationen bereithalten und uns überraschen.

Senninger: Hier gilt natürlich der weise Spruch «en médicine et en amour, ne dis jamais ne dis toujours» (In der Medizin und in der Liebe sage niemals nie und niemals immer). Erkennbar ist aber, dass ein wesentliches Moment in der chirurgischen Technik natürlich die Palpation darstellt. Dies gibt - zumindest derzeit - den entsprechend auch über palpatorische Eindrücke geschulten Chirurgen ein größeres Gefühl der Sicherheit und ist somit verfahrensrelevant. Natürlich lässt sich mit sogenannten Hybrid-Operationen (laparoskopisch assistierte Eingriffe usw.) hier ein Teil dieser Sicherheit auch gestalten. Nicht unerheblich wird aber auch die effektive Operationszeit sein bezüglich der Entscheidung, ob laparoskopisch oder offen operiert werden soll. Wenn eine entsprechende offene Adhäsiolyse innerhalb von einer halben Stunde durchgeführt werden kann, die laparoskopische hingegen 5-6 h benötigt, eventuell in extremer Position des Patienten, treten die gemeinhin gültigen Vorteile laparoskopischer Verfahren deutlich in den Hintergrund.

Frage 3: Im vorliegenden Themenheft sind Neoplasien des Ösophagus, des Magens, des Pankreas und des Kolorektums Gegenstand des Diskurses: Wo ist lhrer Meinung nach die minimalinvasive Methode am sinnvollsten?

Heidecke: Konzeptionell gibt es keine Einschränkungen, am ehesten noch operationstechnischer Natur. Am sinnvollsten in aufsteigender Reihenfolge: Pankreas, Magen/Ösophagus, Kolorektum (vor allem linksseitig).

Keck: Generell denke ich, dass die minimalinvasive Methode dort am sinnvollsten ist, wo durch eine Limitierung des Zugangswegs eine Traumatisierung der Bauchdecke oder des Thorax vermieden werden kann, die allein durch den $\mathrm{Zu}$ gangsweg schon zu einer postoperativen Problematik im Sinne der Mobilisationsfähigkeit des Patienten und der vollen Einsetzbarkeit der Atem- und Bauchmuskulatur führen. Ich denke daher, dass für alle Bereiche der Einsatz von minimalinvasiver Methodik sinnvoll ist. 
In der Wertung des derzeitigen Einsatzes hat sich die laparoskopische Vorgehensweise für Neoplasien des Kolons und Rektums in vielen Kliniken als Standard etabliert. Hier gilt nach den EAES-Konsensus-Guidelines von 2010, dass die laparoskopische Chirurgie für Rektumtumoren im mittleren und unteren Drittel unter optimalen Bedingungen empfohlen werden kann. Diese optimalen Bedingungen beinhalten einen hochspezialisierten Chirurgen, ein hochspezialisiertes Zentrum und selektionierte Patienten unter Ausnahme von T4Tumoren. In der derzeit gültigen S3-Leitlinie zum kolorektalen Karzinom der Arbeitsgemeinschaft der Wissenschaftlichen Medizinischen Fachgesellschaften e.V. (AWMF; 021/007 OL) [3] wird empfohlen, dass die laparoskopische Resektion des Kolon- und Rektumkarzinoms mit entsprechender Expertise des Operateurs und geeigneter Selektion mit gleichen onkologischen Ergebnissen im Vergleich zur offenen Operationstechnik durchgeführt werden kann. Der Empfehlungsgrad A ist begründet auf einem Level of Evidence Ia. Zugrunde liegend sind hierfür die 10-Jahres-Überlebensdaten der britischen CLASICC(Conventional versus Laparoscopic-Assisted Surgery in Colorectal Cancer)-Studie, die die Sicherheit onkologischer Resektionen für die laparoskopische Chirurgie des Kolon-/Rektumkarzinoms belegen konnte [4].

Die laparoskopische Chirurgie des Ösophagus und Magens ist aufgrund einfacher Rekonstruktionstechniken derzeit in vielen Kliniken im Fokus des Interesses. Die laparoskopische Magenchirurgie ist in Korea und Japan für das selektionierte Patientengut von Magenfrühkarzinomen und wenig fortgeschrittenen Magenkarzinomen gut etabliert. Multizentrische, randomisiert kontrollierte Studien zu diesem Thema fehlen. Im Gegensatz zu den asiatischen Ländern ist die Anwendung der laparoskopischen Magenchirurgie in Deutschland bisher nicht weit verbreitet, was erstens in einer höheren Inzidenz fortgeschrittener Magenkarzinome begründet ist und zweitens an den gängigen Empfehlungen der Leitlinie nach AWMF (032-009 OL) liegt, nach der laparoskopische Verfahren zur kurativen Chirurgie des Magenkarzinoms derzeit nicht generell empfohlen werden können.

Die zumindest aus theoretischen Überlegungen größten Vorteile durch ein laparoskopisches Verfahren, was die Reduktion der Zugangswege angeht, hat die thorakoabdominelle Ösophagusresektion. Bei gleichzeitig bestehender einfacher Rekonstruktion bietet sich dieses Verfahren besonders für die Weiterentwicklung minimalinvasiver Technologien auch in der Onkologie an. Auch hier existieren keine prospektiv randomisierten multizentrischen Studien; die Datenlage zur Überlegenheit gegenüber dem konventionellen Vorgehen ist limitiert.

Im Rahmen der onkologischen Pankreaschirurgie kommt zu der komplexen Restriktion erschwerend die komplexe Rekonstruktion hinzu. Diese Komplexität hat bisher dazu geführt, dass komplexe onkologische Resektionen, insbesondere am Pankreaskopf, nur eine limitierte Anwendung gefunden haben. Während in Deutschland daher diese Technik vornehmlich bei selektionierten Patienten Verwendung findet, sind in spezialisierten Zentren in den USA auch fortgeschrittene Pankreastumoren mit Pfortaderresektion vollständig laparoskopisch reseziert worden. Die laparoskopischen Operationen am Pankreaskopf befinden sich derzeit noch in der Evaluation; eine endgültige Wertung der Überlegenheit dieses Verfahrens gegenüber dem konventionellen Vorgehen ist in onkologischen Situationen bisher aufgrund der mangelnden Datenlage nicht möglich.

Lamadé: Dass die minimalinvasive Chirurgie (MIC) bei Karzinomen aller viszeralen Organe einsetzbar und onkologisch korrekt durchführbar ist, konnte bereits gezeigt werden.

Beim kolorektalen Karzinom ist die Laparoskopie Standard in vielen Zentren. Kleinere Tumoren wie gastrointestinale Stromatumoren am Magen sind in MIC-Technik gegebenenfalls in Rendezvousverfahren gefahrlos in geübten Händen durchführbar.

Gastrektomien und Pankreasoperationen sollten auch weiterhin auf hochspezialisierte Zentren beschränkt belieben, da hier die Komplexität und die räumlichen Zugänge sowie die Beurteilung der onkologischen Operabilität allein aufgrund der Lernkurve bei niedrigen Fallzahlen problematisch sein können.

Senninger: Sie ist immer dann am sinnvollsten, wenn sie: i) keine erhebliche Verlängerung der Operation bedeutet; ii) mit gleicher Sicherheit den Status quo sichert und die relevante Anatomie gleich gut darstellt; und iii) im Vergleich zum offenen Vorgehen die gleiche Sicherheit bietet.

Generell gilt hier natürlich, dass die operativen Fähigkeiten des chirurgischen Teams adäquat sind. Die Kostenfrage der in der Regel teureren laparoskopischen Verfahren sollte hier vollkommen im Hintergrund stehen, zumal volkswirtschaftlich zusammengesetzt die laparoskopischen Operationen keineswegs teurer sind.

Frage 4: Welche (theoretischen) Ansätze gibt es, um die bisherigen Limitationen der minimalinvasiven Technik zu überwinden?

Heidecke: Ein Teil der technischen Grenzen ließe sich durch bessere Haptik, bessere Abwinkelbarkeit der Instrumente und Dreidimensionalität des Sehens überwinden. Weiterentwickelte Robotik, Navigation und künstliche Intelligenz würden weiteren Fortschritt bedeuten.

Keck: Insbesondere im Rahmen des Einsatzes robotischer Verfahren existieren nunmehr Technologien, die das laparoskopische Vorgehen konventioneller Art in drei grundlegenden Punkten ergänzen: Dies ist zum Ersten die Dreidimensionalität, zum Zweiten das Operieren unabhängig von Bewegungsartefakten und zum Dritten die dreidimensionale Be- 
weglichkeit von Instrumenten. Diese drei wesentlichen Vorteile der derzeitig verfügbaren robotischen Systeme sind aber nicht notwendigerweise gekoppelt an die derzeitige Master-Slave-Technologie des verfügbaren Da-Vinci-Systems. Zukunftstechnologien müssten die Unwägbarkeiten des sperrigen Master-Slave-Systems mit Konsolenlösung überwinden und die Vorteile Dreidimensionalität, bewegungsartefaktfreies Operieren und dreidimensionale Beweglichkeit laparoskopischer Instrumente vereinen, um hier zukunftsträchtige Technologien für die Weiterentwicklung auch komplexer rekonstruktiver Techniken in der Laparoskopie anzubieten. Insbesondere im Bereich der Dreidimensionalität haben sich hier bereits ausgezeichnete Systeme etabliert, und durch Forschungen im Bereich der dreidimensional beweglichen Instrumente werden weitere Vorteile eines robotischen Vorgehens in Zukunft ausgeglichen werden.

Lamadé: Da die MIC im Gegensatz zur offenen Chirurgie optisch dominiert ist, werden Verfahren der Bildgebung kombiniert mit Navigation bestehende Limitationen überwinden. Fluoreszenzmarkierung von Resektionsgrenzen wäre ein Beispiel solcher intuitiver Navigationstechniken. Fusionen realer Videobilder und 3D geplanter Operationsstrategien kombiniert mit intelligenten Skalpellen, die nur innerhalb definierter räumlicher Pfade schneiden könnten, folgen, sobald die Registrierungsprobleme von Weichgeweben gelöst sind. Die Operationen könnten an personalisierten 3D-Modellen des Patienten noch vor der realen Operation im Simulator durchgespielt werden. Intendierte Bewegungen des Operateurs könnten getriggert durch das Elektroenzephalogramm vom Computer antizipiert und mit der Planung verglichen werden, sodass er nachdrücklich durch Ton- oder Lichtsignale gewarnt werden könnte. Ob allerdings solche Szenarien mit dem Selbstbild des Chirurgen vereinbar sind, ist aber zweifelhaft.

Überraschend ist dann immer noch die taktile und intuitiv wirkende Sicherheit eines erfahrenen Chirurgen, der ohne technische Hilfsmittel Operationen ausführt, die ein Roboter trotz vermeintlich höherer Präzision von hundertstel Millimetern viel schlechter ausführt.

Offensichtlich ist unser angeborener Sensorikapparat, über Jahrmillionen evolutionär optimiert, bisher nur in kleinen Teilen nachstellbar. Trotzdem scheint es bei allem nötigen Respekt dieser Fähigkeiten von Vorteil, dem Chirurgen zusätzliche Informationen in gut erforschter und getesteter Weise anzubieten, ohne zu stören, ohne abzulenken und ohne vorhandene Sinne unnötig zu ersetzen. Hierzu gehört z.B. die Skalierung von Bewegung und die telematische Übertragung der Bewegung auf robotische Hände mit sensorischem Feedback. Keine Übertechnologisierung, keine Überschematisierung. Gute Chirurgie ist einfache Chirurgie.

Senninger: Zunehmende Perfektionierung haptischer Eindrücke; Kombination von präparativen Schritten mit z.B. sonographischer Diagnostik.
Frage 5: Aus Sicht der Patienten: Ist die minimalinvasive Tumorchirurgie ein Pluspunkt?

Heidecke: Aus Sicht des Patienten bezüglich der sekundären Endpunkte (weniger Schmerzen, frühere Rekonvaleszenz, geringere Narben usw.) ja. Bezüglich der onkologischen Endpunkte besteht in der kolorektalen Chirurgie bislang kein Unterschied im Outcome. Dennoch könnte sich das reduzierte operative Trauma positiv auf die Immunsurveillance auswirken und positive Langzeiteffekte haben.

Keck: Aus Sichtweise des Patienten ergeben sich für die laparoskopische Chirurgie folgende, in zahlreichen Studien nachgewiesene Vorteile: Reduktion von Schmerzen, Reduktion von Adhäsionsbeschwerden, Reduktion der postoperativen Atonie, Reduktion der perioperativen Entzündungsreaktionen, verbesserte Lungenfunktion und damit verbunden geringere Krankenhausverweildauer, schnelle Rekonvaleszenz und nicht zuletzt schnellere Arbeitsfähigkeit und verbesserte Kosmetik.

Nachteile laparoskopischer operativer Verfahren sind in der Regel eine verlängerte Operationsdauer, die Lernkurve des Operateurs, teilweise schwierige Präparation sowie hoher technischer und pekuniärer Aufwand. Die Vorteile der minimalinvasiven Tumorchirurgie können nur aus den generellen Vorteilen der laparoskopischen Chirurgie nachvollzogen werden. Alle bisher publizierten Studien, bei zugegebenermaßen bis auf die kolorektale Chirurgie nur geringem Anteil an prospektiv randomisierten Studien, zeigen eine Gleichwertigkeit laparoskopischer Operationsverfahren im Hinblick auf die Onkologie. Ausgenommen von diesen Studien sind in der Regel sehr fortgeschrittene Tumoren, für die der Einsatz auch aus Patientensicht fragwürdig erscheint. Die Vorteile der laparoskopischen Chirurgie, insbesondere auch die Reduktion an Wundinfekten und eine verkürzte Krankenhausverweildauer, suggerieren einen schnelleren Zugang zu adjuvanten Therapiekonzepten und damit auch perspektivisch onkologische Vorteile, die sich für Patienten ergeben könnten.

Lamadé: Aus Sicht der Patienten ist die MIC von überwältigendem Vorteil. So unterscheiden sich die Einschätzungen vor und nach erfolgreichen Operationen. Postoperativ würden sich praktisch alle Patienten wieder minimalinvasiv operieren lassen oder sogar noch weniger invasiven Maßnahmen zustimmen, obwohl vor Operationen abhängig vom eigenen Körperselbstbild normalerweise nur der medizinische Vorteil als Entscheidung für oder gegen die MIC gesehen wird.

Da Rekonvaleszenz und Komplikationen wahrscheinlich langfristig niedriger sein werden, wird sich diese Entwicklung zugunsten der MIC entscheiden.

Senninger: Auf jeden Fall, zumindest im Bereich der Diagnostik und der potenziell kurativen Operation bei früheren 
Tumorstadien. Zum jetzigen Zeitpunkt würden wir aber bei einem fortgeschrittenen Malignom - mit Ausnahme der diagnostischen Schritte - die Operation weiterhin offen durchführen.

\section{Frage 6: Beeinflusst die minimalinvasive Technik die Stellung der onkologischen Chirurgie in der multimodalen Krebsbehandlung?}

Heidecke: Nein, die onkologischen Prinzipien (R0-Resektion, adäquate Lymphadenektomie) müssen eingehalten werden. Ferner müssen die Rekonstruktionsmöglichkeiten gleichermaßen sicher sein.

Keck: Dies ist eine wichtige Frage. Eine aktuelle Metaanalyse von Biagi et al. [5] zeigt, dass in der Metaanalyse von neun Kohortenstudien der Zeitpunkt des Einsetzens der adjuvanten Therapie des kolorektalen Karzinoms erheblichen Einfluss auf die Mortalität hat. Für jede Verlängerung von 4 Wochen zeigt sich in dieser Analyse eine Erhöhung des Mortalitätsrisikos um 14\%. Nach dieser Analyse suggerieren minimalinvasive Techniken, die erwiesenermaßen weniger Wundinfekte und damit eine kürzere Hospitalisationsdauer haben, den frühen und zeitgerechten Zugang zur Chemotherapie. Perspektivisch können so minimalinvasive Techniken auch den Stellenwert der onkologischen Chirurgie in multimodalen Therapieansätzen erheblich beeinflussen.

Lamadé: Ohne große Beeinflussung systemischer Therapien können Staging-Laparoskopien und Tumorgewebegewinnung über MIC-Techniken erfolgen. Ein Zeitverlust bis zum Beginn z.B. einer Chemotherapie kann vermieden werden. Das Platzieren von Brachytherapie-Systemen vereinfacht sich. Bridging-Konzepte können durch MIC erst andere systemische Maßnahmen möglich machen.
Senninger: Es handelt sich hierbei um eine Methode, die bei entsprechender Befähigung des operativen Teams die gleichen guten oder schlechten Ergebnisse wie die offene Operationstechnik liefert. Es sollte somit unter Hinweis auf die (vermeintlich!) minimalinvasive Technik die prinzipielle Operationsindikation überhaupt nicht beeinflusst werden.

Dennoch wird unter Umständen ein Patientenkollektiv, das multimorbide ist und von einer minimalinvasiven Technik profitieren kann, möglicherweise doch häufiger auch einem operativen Teilschritt unterzogen. Hierbei fehlt aber zumeist eine eindeutige wissenschaftliche Beweisführung mit hoher Evidenz.

\section{Teilnehmer}

Prof. Dr. med. Claus-Dieter Heidecke

Abteilung für Allgemeine Chirurgie, Viszeral-, Thorax- und Gefäßchirurgie

Klinik und Poliklinik für Chirurgie, Universitätsmedizin Greifswald Ferdinand-Sauerbruch-Straße, 17475 Greifswald, Deutschland heidecke@uni-greifswald.de

Prof. Dr. med. Tobias Keck

Klinik für Chirurgie

Universitätsklinikum Schleswig-Holstein, Campus Lübeck

Ratzeburger Allee 160, 23538 Lübeck, Deutschland

tobias.keck@uksh.de

Prof. Dr. med. Wolfram Lamadé

Allgemein- und Viszeralchirurgie

HELIOS Privatklinik Überlingen

Härlenweg 1, 88662 Überlingen, Deutschland

wolfram.lamade@helios-kliniken.de

Univ.-Prof. Dr. med. Dr. h.c. Norbert Senninger, FACS, FRCS

Klinik für Allgemein- und Viszeralchirurgie

Universitätsklinikum Münster

Albert-Schweitzer-Campus 1, Gebäude W1

Waldeyerstraße 1, 48149 Münster, Deutschland

senning@uni-muenster.de

\section{Literatur}

1 Lee SW, Southall J, Allendorf J, Bessler M, Whelan RL: Traumatic handling of the tumor independent of pneumoperitoneum increases port site implantation rate of colon cancer in a murine model. Surg Endosc 1998;12:828-834.

- Wittich P, Marquet RL, Kazemier G, Bonjer HJ Port-site metastases after $\mathrm{CO}_{2}$ laparoscopy. Is aerosolization of tumor cells a pivotal factor? Surg Endosc 2000;14:189-192.
3 Leitlinienprogramm Onkologie (Deutsche Krebsgesellschaft, Deutsche Krebshilfe, AWMF): S3Leitlinie Kolorektales Karzinom, Langversion 1.0 - Juni 2013. AWMF-Registernummer: 021/007OL. www.awmf.org/leitlinien/detail/ll/021-007OL.html.

4 Green BL, Marshall HC, Collinson F, Quirke P, Guillou P, Jayne DG, Brown JM: Long-term follow-up of the Medical Research Council CLASICC trial of conventional versus laparoscopically assisted resection in colorectal cancer. $\mathrm{Br} \mathrm{J}$ Surg 2013;100:75-82.
5 Biagi JJ, Raphael MJ, Mackillop WJ, Kong W, King WD, Booth CM: Association between time to initiation of adjuvant chemotherapy and survival in colorectal cancer: a systematic review and metaanalysis. JAMA 2011;305:2335-2342. 